\title{
Selective Production of Rydberg-Stark States of Positronium
}

\author{
T. E. Wall, A. M. Alonso, B. S. Cooper, A. Deller, S. D. Hogan, and D. B. Cassidy \\ Department of Physics and Astronomy, University College London, Gower Street, London WC1E 6BT, United Kingdom
}

(Received 4 February 2015; published 28 April 2015)

\begin{abstract}
Rydberg positronium (Ps) atoms have been prepared in selected Stark states via two-step $(1 s \rightarrow 2 p \rightarrow n d / n s)$ optical excitation. Two methods have been used to achieve Stark-state selection: a field ionization filter that transmits the outermost states with positive Stark shifts, and state-selected photoexcitation in a strong electric field. The former is demonstrated for $n=17$ and 18 while the latter is performed for $n=11$ in a homogeneous electric field of $1.9 \mathrm{kV} / \mathrm{cm}$. The observed spectral intensities and their dependence on the polarization of the laser radiation are in agreement with calculations that include the perturbations of the intermediate $n=2$ manifold. Our results pave the way for the generation of Rydberg Ps atoms with large electric dipole moments that are required for the realization of schemes to control their motion using inhomogeneous electric fields, an essential feature of some proposed Ps free-fall measurements requiring focused beams of long-lived atoms.
\end{abstract}

DOI: 10.1103/PhysRevLett.114.173001

PACS numbers: $32.80 . \mathrm{Rm}, 36.10 . \mathrm{Dr}, 78.70 . \mathrm{Bj}$

The concept of using inhomogeneous electric fields to manipulate the motion of electrically neutral atomic or molecular systems that possess nonzero electric dipole moments has been around for many years [1]. Applying such methods to atoms and molecules in high Rydberg states, which have large electric dipole moments, allows strong forces to be generated, facilitating exceptional control [2-4]. In this way atom-optics elements for neutral atoms may be constructed, including electrostatic mirrors [5], lenses [6], and three-dimensional traps [7,8].

It would be particularly advantageous to use similar methods to control positronium (Ps) atoms because the manner in which they are created invariably leads to energetic and divergent beams (e.g., Ref. [9]). Moreover, because it is composed of a particle-antiparticle pair Ps is intrinsically unstable, and ground state triplet atoms selfannihilate with a mean lifetime of $142 \mathrm{~ns}$ [10]. Ps decay can be avoided, however, by excitation into Rydberg states for which annihilation is negligible. The use of electric fields for deceleration and focussing of Rydberg Ps atoms would enable a wide range of experiments, from spectroscopic studies to gravity measurements.

For Rydberg Ps atoms with radiative lifetimes significantly greater than $142 \mathrm{~ns}$, annihilation is no longer a defining characteristic of the system, and these atoms can be thought of simply as very low mass $\left(M=2 m_{e}\right)$ hydrogenic atoms. There is, therefore, no fundamental reason why one cannot use inhomogeneous electric fields to control Rydberg Ps atoms in the same way as has been demonstrated for

Published by the American Physical Society under the terms of the Creative Commons Attribution 3.0 License. Further distribution of this work must maintain attribution to the author(s) and the published article's title, journal citation, and DOI. hydrogen [11] and other atoms and molecules. In fact, Ps is ideally suited to manipulation in this way; the kinetic energy that has to be extracted from a thermal Ps atom to bring it to rest $(25 \mathrm{meV})$ is comparable to what has already been demonstrated (40 meV) [12]. The application of these methods to Ps has not yet been realized, even though Rydberg Ps was first generated in the laboratory more than two decades ago [13]. In that work the efficiency with which Rydberg atoms were created and detected was low, and it was possible only to demonstrate the production of a few highlying states $(n=13-15)$. More recently positron trapping techniques [14] have been used to generate Rydberg Ps much more efficiently [15], making further experimentation feasible. There are, for example, experiments currently underway to use Rydberg Ps atoms to produce antihydrogen $[16,17]$, create electron-positron plasmas [18], and perform precision, Doppler-corrected, Ps spectroscopy [19]. The ultimate goal of the present work is to make a focussed Ps beam suitable for gravity measurements [20].

The ability to selectively populate individual RydbergStark states is a prerequisite for many schemes designed to control Ps atoms via their electric dipole moments. Achieving this goal with Ps is especially challenging because the production mechanism generally leads to atoms that are few in number, spread out spatially, fastmoving, and close to a solid surface, all of which are detrimental to spectroscopic investigations in homogeneous electric fields. We describe here experiments in which these obstacles are addressed, and Ps atoms are excited to selected Rydberg-Stark states. We use the same $1 s \rightarrow 2 p \rightarrow n d / n s$ two-step excitation scheme demonstrated previously $[13,15,19,21]$, except in this case the infrared (IR) laser used to drive the $2 p \rightarrow n d / n s$ transitions has a narrower bandwidth of $\sim 5 \mathrm{GHz}$, while the bandwidth of the ultraviolet (UV) laser is $\sim 85 \mathrm{GHz}$. 
The positron beam and trap system used here is similar to that described elsewhere [22]. Positrons are accumulated for $1 \mathrm{~s}$ in the trap in an axial magnetic field of $\sim 600 \mathrm{G}$, and then implanted into the target in a 4 ns wide pulse with a diameter of $2.5 \mathrm{~mm}$. Each pulse contains $\sim 5 \times 10^{5}$ positrons, of which $230 \%$ form Ps atoms.

The target used in this work was a porous silica film [23] which, following irradiation with $\mathrm{keV}$ positrons, emits ground-state Ps atoms into vacuum with near thermal energies [24]. When Ps atoms annihilate, either by selfannihilation or following collisions with electrodes or the vacuum chamber, they emit gamma radiation, with photon energies $\leq 511 \mathrm{keV}$ [10]. The time dependence of this emission can be used to monitor the production of Ps atoms, and subsequent laser excitation thereof, using single-shot positron annihilation lifetime spectroscopy (SSPALS) [25]. The gamma ray detector used here was a lead tungstate scintillator attached to a photomultiplier tube [26]. It was located $\sim 4 \mathrm{~cm}$ from the silica target, such that long-lived Ps atoms moved towards it (see Fig. 1). The SSPALS annihilation signal is characterized using the fraction, $f$,

$$
f=\int_{B}^{C} V(t) d t / \int_{A}^{C} V(t) d t
$$

where $V(t)$ refers to the time-dependent voltage output from the detector. The integration regions are chosen depending on the processes that are to be studied, and are defined relative to the positron pulse implantation time. We characterize laser-induced effects using the parameter $S=\left(f_{\mathrm{bk}}-f_{\mathrm{sig}}\right) / f_{\mathrm{bk}}$, where $f_{\mathrm{bk}}$ refers to background measurements [with the laser(s) tuned off resonance] and $f_{\text {sig }}$ to a measurement made with the lasers set up to drive the transitions to be studied.
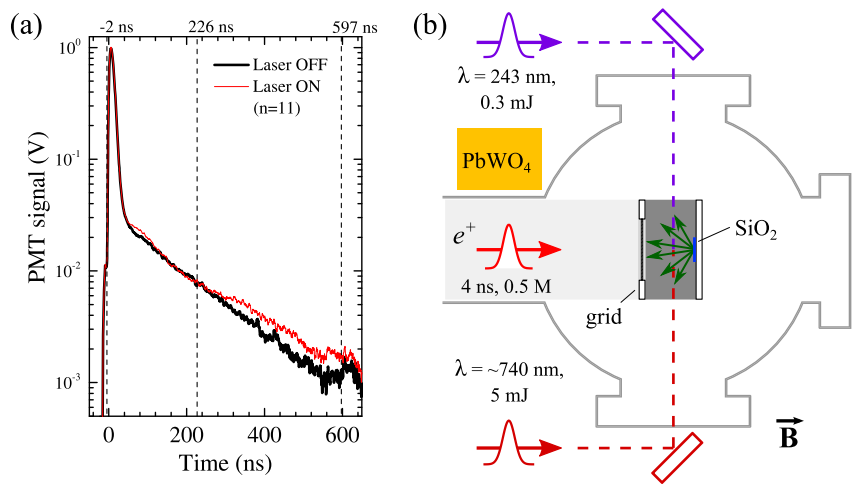

FIG. 1 (color online). (a) Example of single shot lifetime spectra with lasers on and off resonance as indicated, and (b) a schematic of the target chamber and Ps formation region. The time windows used to analyze the lifetime spectra are indicated by the dashed lines in (a). The shaded regions in (b) roughly correspond to annihilation times up to $226 \mathrm{~ns}$ (dark region) and later than $226 \mathrm{~ns}$ (light region).
The silica target was mounted $8 \mathrm{~mm}$ behind a transmission grid (nominal open area 70\%), allowing independent control of the positron beam implantation energy and the electric field in the excitation region. This made it possible to conduct experiments in arbitrary electric fields without affecting the ground state Ps properties. For typical Ps velocities the mean time to travel from the silica target to the grid was $\sim 100 \mathrm{~ns}$, but due to the Ps velocity and angular distributions the flight-time distribution downstream is rather broad. However, all of the Rydberg states produced in this work have lifetimes exceeding $1 \mu$ s (e.g., Ref. [20]) and will therefore live long enough to reach the grid. The target layout is shown in Fig. 1, along with examples of single shot lifetime spectra with the laser on and off resonance. The former shows an excess of gamma radiation at longer times, which gives rise to negative $S$ values using the analysis described above. Also visible are annihilations at earlier times due to Ps atoms colliding with the grid.

Rydberg Ps atoms were prepared using a two-step excitation scheme $[13,15,19]$, driven by the output of two pulsed dye lasers pumped by the same Nd:YAG laser (pulse width $7 \mathrm{~ns}$ ). The $1 s \rightarrow 2 p$ transition was driven using $\sim 0.5 \mathrm{~mJ}$ of $243 \mathrm{~nm}$ light, while $2 p \rightarrow n d / n s$ transitions were driven using IR light, tunable from 729-769 nm. An IR fluence of $\sim 2 \mathrm{~mJ} / \mathrm{cm}^{2}$ was found to be sufficient to saturate the $2 p \rightarrow 11 d$ transition. The $\sim 90 \mathrm{GHz}$ spectral resolution of our measurement was dominated by the bandwidth of the UV laser, and for scans conducted in strong electric fields the Stark-broadened $n=2$ manifold must also be considered [27].

The polarization of both laser beams was selected using $\lambda / 2$ plates, producing linearly polarized light with $>95 \%$ purity. Light with horizontal (vertical) polarization, parallel (perpendicular) to the electric field in the photoexcitation region, drives transitions for which $\Delta M_{J}=0$, $\left(\Delta M_{J}= \pm 1\right)$. The production of Ps Rydberg states ranging from $n=9$ up to the ionization limit was verified in measurements conducted in (nominally) zero electric field, as shown in Fig. 2. The states were resolved up to $n \sim 30$ [Fig. 2(c)]. For these measurements the background signal $f_{\text {bk }}$ was obtained with the UV laser tuned to the $1 s \rightarrow 2 p$ resonance, and the average of measurements made with the IR laser scanned from $755-758.5 \mathrm{~nm}$, so that $S$ is sensitive only to the $2 p \rightarrow n d / n s$ transitions. The nonzero background level for $\lambda>760 \mathrm{~nm}$ [Fig. 2(a)] is due to the production of Rydberg states by broadband light emitted via amplified spontaneous emission (e.g., Ref. [28]), as these wavelengths approach the upper limit of the gain curve of the Styryl-8 dye used in the IR laser. Both lasers were linearly polarized in the vertical direction for these measurements.

A notable feature of these data is that the negative $S$ values are inverted for $n>17$, becoming positive thereafter. The timing windows used to analyze these data are such that this inversion occurs when Ps atoms are no longer 


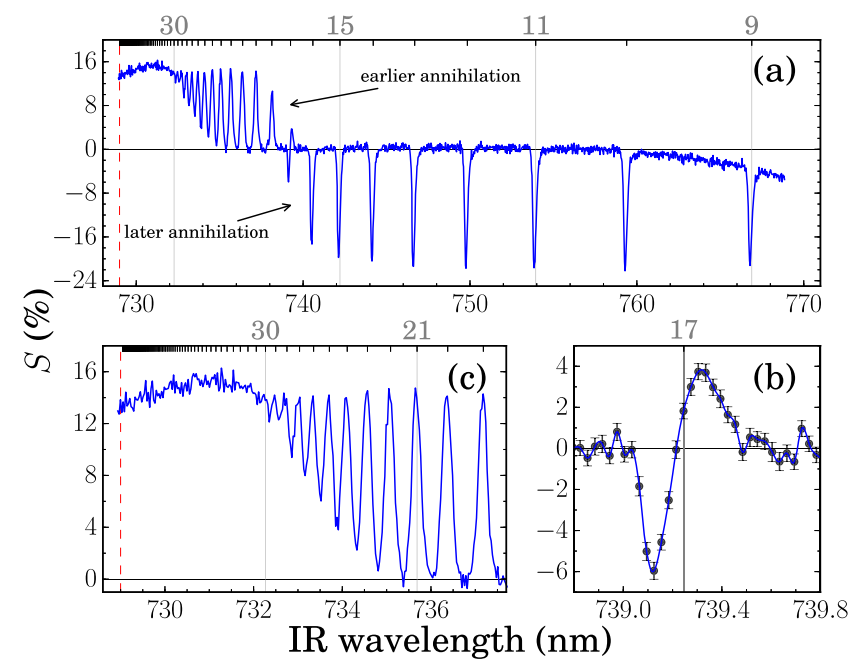

FIG. 2 (color online). (a) Spectrum of Rydberg states excited in zero applied electric field. The data for $n=17$ are expanded in (b) and $n \geq 19$ is shown in (c). The reversal of the sign of $S$ is due to Ps atoms that are field ionized near the grid (see text). The dashed lines indicate the ionization limit $(729 \mathrm{~nm})$. The timing windows used to analyze these data were $A=-2 \mathrm{~ns}$, $B=226 \mathrm{~ns}$, and $C=597 \mathrm{~ns})$. The error bars, not shown in (a) or (c), are of the same size as those shown in (b). Values of $n$ are shown in the top axes.

able to move beyond the grid due to field ionization. When this occurs liberated positrons will return to the target in less than $1 \mathrm{~ns}$, where they will mostly annihilate, giving rise to an annihilation signal at earlier times. We note that the magnitude of the positive and negative peaks cannot be directly compared as the detection efficiency is not the same in both cases. No electric field was applied in the excitation region when these data were recorded; however, the $100 \mathrm{G}$ magnetic field will give rise to induced fields $\lesssim 10 \mathrm{~V} / \mathrm{cm}$. There may also be fields generated by secondary electrons produced by positron irradiation of the target [29] or photoelectrons made by the UV laser. The combined effect of these fields polarizes the Rydberg states in the excitation region, leading to a weak Stark splitting [30].

As the Rydberg Ps atoms pass through the grid they experience a large inhomogeneous electric field on a distance scale commensurate with the mesh spacing $(\sim 100 \mu \mathrm{m})$. After being transmitted any remaining Rydberg Ps atoms experience an approximately homogeneous electric field defined by the potential applied to the grid plane. The data presented in Fig. 3 show spectra covering the transition to the $n=18$ state, and demonstrate that transmission of Rydberg Ps atoms through the grid depends on their rate of electric field ionization. These data were recorded with a constant electric field of $63 \mathrm{~V} / \mathrm{cm}$ applied to the excitation region and fields ranging from 1397 to $1985 \mathrm{~V} / \mathrm{cm}$ in the region after the grid.

For weak fields, the short wavelength components of the $n=18$ spectral feature are transmitted through the grid (negative $S$ parameter) while only the outermost

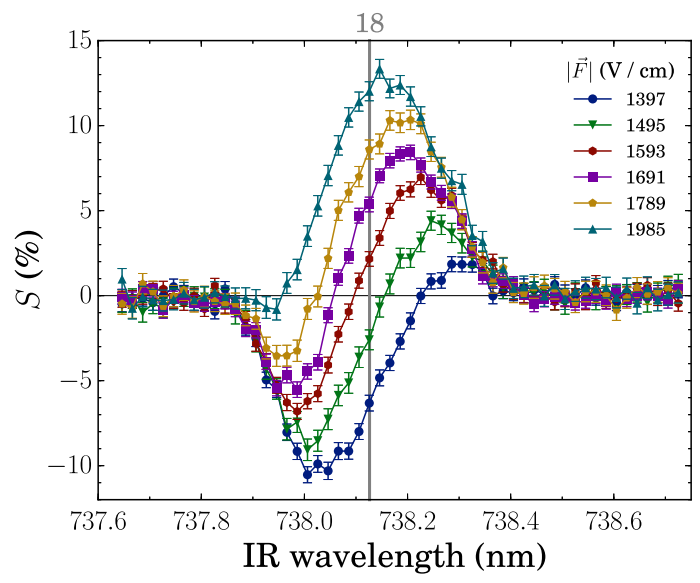

FIG. 3 (color online). Transitions to $n=18$ in a $63 \mathrm{~V} / \mathrm{cm}$ electric field in the excitation region and fields outside the grid as indicated. The vertical line shows the position of the field free peak. Positive $S$ values indicate atoms in Stark states that cannot pass beyond the grid due to field ionization.

components at the long wavelength edge are not transmitted. This observation indicates that Rydberg-Stark states are sufficiently polarized by the electric field to result in a partial splitting of states with positive and negative Stark shifts, and that these states subsequently evolve adiabatically while passing from the excitation region through the grid.

Since the outermost Stark states with negative Stark shifts ionize in weaker electric fields than states with positive Stark shifts [31], the cutoff in transmission through the grid occurs first for the outermost components at the long wavelength edge of the spectrum. For each value of $n$, the ionization field for the outermost Stark state with a negative Stark shift in Ps is approximately equal to the classical ionization field [30] $F_{\text {ion }}=2 R_{\mathrm{Ps}} h c / e a_{P s} 9 n^{4}$, where $R_{\mathrm{Ps}}=0.5 R_{\infty}$ is the Rydberg constant for Ps, and $a_{\mathrm{Ps}}=2 a_{0}=1.058 \times 10^{-10} \mathrm{~m}$ is the Ps Bohr radius. This suggests an ionization field of $1360 \mathrm{~V} / \mathrm{cm}$ for $n=18$, close to that observed for the transmission of the corresponding states in Fig. 3. On the other hand, the outermost Stark states with positive Stark shifts ionize at approximately $2 F_{\text {ion }}$, indicating that a field of $2721 \mathrm{~V} / \mathrm{cm}$ is required to completely inhibit transmission through the grid for all states with $n=18$. The observation that a field slightly below this is sufficient to inhibit transmission of the outermost states with positive Stark shifts can be attributed to effects of $n$-mixing with states of higher principal quantum number in the combined magnetic and inhomogeneous electric fields in the vicinity of the grid. In this configuration the grid acts as a filter for the unresolved Rydberg-Stark states. This Stark-state filter permits spectral broadening arising from electric fields to be distinguished from Doppler broadening. This makes it possible to select particular Stark states even if Doppler broadening is very large, and to obtain information on the magnitude of the 
electric field in the photoexcitation region. The states that are not field ionized may still experience forces due to field gradients and be deflected; it remains to be seen exactly how the trajectories of transmitted atoms are affected by the grid and applied fields, and this will be the subject of future experiments in which Ps Rydberg atoms will be directly imaged.

To demonstrate the possibility of resolving individual Rydberg-Stark states, the spectra presented in Fig. 4 were recorded at $n=11$ in an electric field of $1.9 \mathrm{kV} / \mathrm{cm}$. In this field, individual Stark states with $n=11$ and equal values of the azimuthal quantum number $\left|m_{\ell}\right|$, are separated in frequency by $\sim 165 \mathrm{GHz}$. However, this field also gives rise to mixing between the intermediate $2^{3} S$ and $2^{3} P$ terms, and unresolved Stark splittings at $n=2$ approaching $30 \mathrm{GHz}$. The complexity of the Stark structure arising from the spinorbit coupling in this intermediate state means that the Stark manifolds in the Rydberg states cannot be fully described

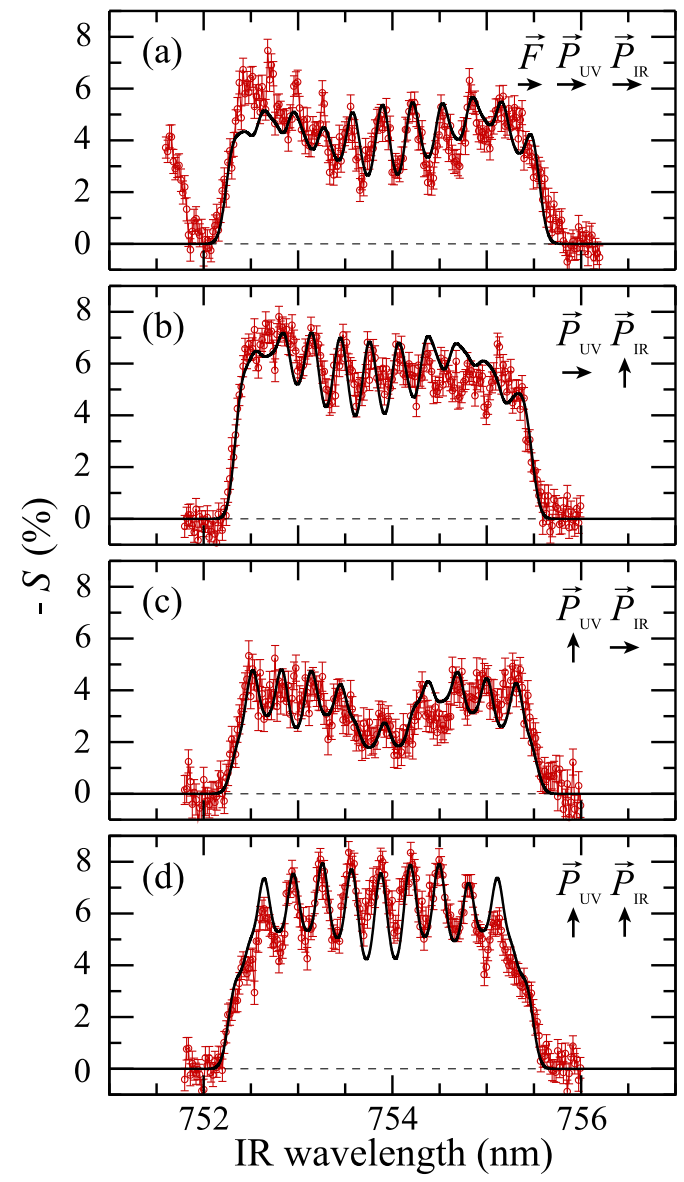

FIG. 4 (color online). Measured (points) and calculated (lines) Rydberg-Stark spectra of $n=11$ Ps atoms in an electric field of $1.9 \mathrm{kV} / \mathrm{cm}$. The excitation was performed with four different combinations of laser polarizations $P$ with respect to the electric field direction $F$, as indicated in (a)-(d). In panel (a) the intrusion of neighboring $n=12$ states (not included in the calculation) is seen below $752 \mathrm{~nm}$. by hydrogenic expressions for individual values of $\left|m_{\ell}\right|$, even at the $90 \mathrm{GHz}$ resolution of the experiment. These effects are accounted for by calculating the eigenvalues and eigenvectors of the Hamiltonian matrix including the electric field for the triplet states of Ps with $n=2$ in an $\left|n S \ell J M_{J}\right\rangle$ basis [27] to determine the relative populations of each Stark sublevel. The results of this calculation were then used to determine the intensities of the subsequent transitions to the $11 s, 11 p$, and $11 d$ Rydberg-Stark states following a transformation to the $\left|n \ell m_{\ell}\right\rangle$ basis for the description of these states. The results of this calculation, including a convolution with a $90 \mathrm{GHz}$ FWHM Gaussian spectral function, are overlaid with the experimental data in Fig. 4.

The agreement between the calculation and the measurement was achieved with only one free parameter, the spectral intensity at which saturation occurs, $\sim 10^{-5} e^{2} a_{\mathrm{Ps}}^{2}$. This was determined using a simple fitting routine for each spectrum and is similar to that found in previous measurements [32]. Better agreement can be obtained by modifying the relative $\left|m_{\ell}\right|$ populations in the Rydberg states, but the present approach is sufficient to highlight the underlying physical mechanisms. The calculation does not account for the polarization of the positron beam or optical pumping through $1 S \leftrightarrow 2 P$ transitions. The Rydberg-Stark states at the edges of the spectra are those with the largest electric dipole moments of $\sim 165 e a_{\mathrm{Ps}} \simeq 840 \mathrm{D}$. They are therefore most sensitive to inhomogeneities in the electric fields in the photoexcitation region, which can give rise to broadening of the associated spectral features. The asymmetric structures observed in the spectra of Fig. 4 result primarily from the Stark structure of the $n=2$ manifold.

The data presented here represent the first observation of selected Ps Rydberg-Stark states, which is a crucial step towards developing the atom optics [20] required for a Ps gravity experiment [33]. The Stark filter constitutes a rudimentary manipulation of Ps Rydberg-Stark states of differing dipole moments, an area of study that will be expanded in future work. By generating well-defined electric field distributions and imaging Rydberg Ps atoms we will implement schemes similar to those used previously for other atoms [5,7], including the intriguing possibility of using a wire to capture high-field seeking states into circular orbits [34].

Focusing, deceleration, and other manipulations of Ps motion and velocities will open the door to a new era of Ps experimentation, with immediate application to precision Ps spectroscopy measurements [35], for which second order Doppler effects are a limiting factor [36]. Future refinements of the positron beam will make it possible to use a Doppler-free two-photon excitation scheme [37], providing improved resolution and state selectivity at higher values of $n$. While this may benefit eventual Ps gravity measurements [20], many important initial experiments can be realized using the present methodology, 
including testing schemes to generate very long-lived circular Ps states [38-42].

The authors are grateful to L. Liszkay for providing silica samples. This work was supported by UCL through its Impact Studentship Programme, and was funded in part by the Leverhulme trust (Grant No. RPG-2013-055), the ERC (Grant No. CIG 630119) and the EPSRC (Grant No. EP/K028774/1).

[1] H. G. Bennewitz, W. Paul, and C. Schlier, Z. Phys. 141, 6 (1955).

[2] W. H. Wing, Phys. Rev. Lett. 45, 631 (1980).

[3] T. Breeden and H. Metcalf, Phys. Rev. Lett. 47, 1726 (1981).

[4] D. Townsend, A. L. Goodgame, S. R. Procter, S. R. Mackenzie, and T. P. Softley, J. Phys. B 34, 439 (2001).

[5] E. Vliegen and F. Merkt, Phys. Rev. Lett. 97, 033002 (2006).

[6] E. Vliegen, P. A. Limacher, and F. Merkt, Eur. Phys. J. D 40, 73 (2006).

[7] S. D. Hogan and F. Merkt, Phys. Rev. Lett. 100, 043001 (2008).

[8] S. D. Hogan, C. Seiler, and F. Merkt, Phys. Rev. Lett. 103, 123001 (2009).

[9] P. J. Schultz and K. G. Lynn, Rev. Mod. Phys. 60, 701 (1988).

[10] A. Rich, Rev. Mod. Phys. 53, 127 (1981).

[11] E. Vliegen, S. D. Hogan, H. Schmutz, and F. Merkt, Phys. Rev. A 76, 023405 (2007).

[12] P. Lancuba and S. D. Hogan, Phys. Rev. A 90, 053420 (2014).

[13] K. P. Ziock, R. H. Howell, F. Magnotta, R. A. Failor, and K. M. Jones, Phys. Rev. Lett. 64, 2366 (1990).

[14] J. R. Danielson, D. H. E. Dubin, R. G. Greaves, and C. M. Surko, Rev. Mod. Phys. 87, 247 (2015).

[15] D. B. Cassidy, T. H. Hisakado, H. W. K. Tom, and A. P. Mills, Phys. Rev. Lett. 108, 043401 (2012).

[16] A. Kellerbauer et al., Nucl. Instrum. Methods Phys. Res., Sect. B 266, 351 (2008).

[17] P. Perez and Y. Sacquin, Class. Quantum Grav. 29, 184008 (2012).

[18] T. S. Pedersen, J. R. Danielson, C. Hugenschmidt, G. Marx, X. Sarasola, F. Schauer, L. Schweikhard, C. M. Surko, and E. Winkler, New J. Phys. 14, 035010 (2012).
[19] A. C. L. Jones, T. H. Hisakado, H. J. Goldman, H. W. K. Tom, A. P. Mills, and D. B. Cassidy, Phys. Rev. A 90, 012503 (2014).

[20] D. B. Cassidy and S. D. Hogan, Int. J. Mod. Phys. Conf. Ser. 30, 1460259 (2014).

[21] K. P. Ziock, C. D. Dermer, R. H. Howell, F. Magnotta, and K. M. Jones, J. Phys. B 23, 329 (1990).

[22] D. B. Cassidy, S. H. M. Deng, R. G. Greaves, and A. P. Mills, Jr., Rev. Sci. Instrum. 77, 073106 (2006).

[23] L. Liszkay, F. Guillemot, C. Corbel, J.-P. Boilot, T. Gacoin, E. Barthel, P. Prez, M.-F. Barthe, P. Desgardin, P. Crivelli, U. Gendotti, and A. Rubbia, New J. Phys. 14, 065009 (2012).

[24] P. Crivelli, U. Gendotti, A. Rubbia, L. Liszkay, P. Perez, and C. Corbel, Phys. Rev. A 81, 052703 (2010).

[25] D. B. Cassidy, S. H. M. Deng, H. K. M. Tanaka, and A. P. Mills, Jr., Appl. Phys. Lett. 88, 194105 (2006).

[26] D. B. Cassidy and A. P. Mills, Jr., Nucl. Instrum. Methods Phys. Res., Sect. A 580, 1338 (2007).

[27] S. M. Curry, Phys. Rev. A 7, 447 (1973).

[28] W. Demtröder, Laser Spectroscopy, 3rd ed. (Springer, New York, 2003).

[29] N. Overton and P. G. Coleman, Phys. Rev. Lett. 79, 305 (1997).

[30] T.F. Gallagher, Rydberg Atoms (Cambridge University Press, Cambridge, England, 1994).

[31] R. J. Damburg and V. V. Kolosov, J. Phys. B 12, 2637 (1979).

[32] S. D. Hogan, Phys. Rev. A 87, 063423 (2013).

[33] A. P. Mills and M. Leventhal, Nucl. Instrum. Methods Phys. Res., Sect. B 192, 102 (2002).

[34] H. Ko and S. D. Hogan, Phys. Rev. A 89, 053410 (2014).

[35] P. Crivelli, C. L. Cesar, and U. Gendotti, Can. J. Phys.89, 29 (2011).

[36] M. S. Fee, A. P. Mills, S. Chu, E. D. Shaw, K. Danzmann, R. J. Chichester, and D. M. Zuckerman, Phys. Rev. Lett. 70, 1397 (1993).

[37] T. E. Wall, D. B. Cassidy, and S. D. Hogan, Phys. Rev. A 90, 053430 (2014).

[38] R. G. Hulet and D. Kleppner, Phys. Rev. Lett. 51, 1430 (1983).

[39] D. Delande and J. C. Gay, Europhys. Lett. 5, 303 (1988).

[40] J. Hare, M. Gross, and P. Goy, Phys. Rev. Lett. 61, 1938 (1988).

[41] C. H. Cheng, C. Y. Lee, and T. F. Gallagher, Phys. Rev. Lett. 73, 3078 (1994).

[42] R. Lutwak, J. Holley, P. P. Chang, S. Paine, D. Kleppner, and T. Ducas, Phys. Rev. A 56, 1443 (1997). 